\title{
Influence des facteurs héliothermiques et nutritionnels sur la croissance et les capacités de stockage intracellulaire (azote et phosphore) chez Microcystis aeruginosa Kützing isolée de la retenue eutrophe El Kansera (Maroc)
}

\author{
L. Boumnich ${ }^{1}$ \\ M. Derraz ${ }^{2}$ \\ B. $\mathrm{Naji}^{2}$ \\ A. Dauta ${ }^{3}$
}

Mots-clés : Microcystis aeruginosa, taux de croissance, lumière, température, réserves intracellulaires, chlorophylle a, azote, phosphore.

L'interdépendance de la lumière et de la température a été étudiée, sur une culture non axénique de Microcystis aeruginosa, isolée de la retenue eutrophe $\mathrm{El} \mathrm{Kansera.} \mathrm{Les} \mathrm{expériences} \mathrm{ont} \mathrm{été} \mathrm{réalisées} \mathrm{dans} \mathrm{une} \mathrm{plage} \mathrm{de} \mathrm{température} \mathrm{de} 15$ à $30^{\circ} \mathrm{C}$ et des lumières comprises entre. 5 et $500 \mu \mathrm{E} . \mathrm{m}^{-2} \cdot \mathrm{s}^{-1}$, sous une photopériode de $15 / 9$. Le taux de croissance maximal $\left(\mu \max =0.92 \mathrm{j}^{-1}\right)$ a été obtenu, en culture batch sans bullage, sous une température de $20^{\circ} \mathrm{C}\left( \pm 1^{\circ} \mathrm{C}\right)$ et une lumière optimale (Iopt) de $115 \mu \mathrm{E} . \mathrm{m}^{-2} \cdot \mathrm{s}^{-1}$.

Le suivi de la culture de cette espèce, sous des conditions de lumière et de température suboptimales et dans des conditions non limitantes en nutriments, montre que les deux paramètres utilisés pour l'estimation de la biomasse (chlorophylle a et densité optique) présentent une bonne corrélation $\left(r^{2}=0.98\right)$. Une carence en nutriments $(N, P)$ induit une diminution des teneurs en pigments cellulaires, beaucoup plus marquée dans le cas de l'azote.

Les réserves intracellulaires et les capacités de stockage des nutriments $\mathrm{N}$ et $\mathrm{P}$ (quotas internes en azote et phosphore) ont été déterminées expérimentalement. Les résultats obtenus montrent que cette cyanobactérie est capable de stocker beaucoup plus de phosphore que d'azote et que les teneurs en chlorophylle a évoluent en relation avec le niveau de carence.

Influence of light-temperature and nutrient conditions on the growth and intracellular storage (Nitrogen and Phosphorus) of Microcystis aeruginosa Kützing isolated from the El Kansera (Morocco) eutrophic impoundment

Keywords : Microcystis aeruginosa, growth rate, light, temperature, intracellular stock, chlorophyll a, nitrogen, phosphorus.

The interdependence between light and temperature was studied in a non axenic culture of Microcystis aeruginosa, isolated from the El Kansera eutrophic impoundment. The experiments were performed, in batch culture without bubbling, over a range of light intensities $5-500 \mu \mathrm{E} . \mathrm{m}^{-2} \mathrm{~s}^{-1}$ provided by $400 \mathrm{~W}$ lamps (phytoclaude) under $15 / 9$ light : dark cycles at various temperature from 15 to $30^{\circ} \mathrm{C}$. The maximum growth rate $\left(\mu \max =0.92\right.$ day $\left.^{-1}\right)$ was recorded for growth conditions at $20^{\circ} \mathrm{C}\left( \pm 1^{\circ} \mathrm{C}\right)$ and a light intensity (Iopt) of $115 \mu \mathrm{E} . \mathrm{m}^{-2} \cdot \mathrm{s}^{-1}$.

Under non limited nutrient conditions and for suboptimal temperature, a good relationship was found $\left(\mathrm{r}^{2}=0.98\right)$ between optical density $(750 \mathrm{~nm})$ and the chlorophyll a values. In case of nutrient deficiency, the pigment content was more affected by nitrogen starvation.

Intracellular and cell storage capacity were deduced from experiments. This strain is able to store high quantities of nutrients, and the chlorophyll a content varyies according to the degree of starvation.

1. Université Chouaib Doukkali, Faculté des sciences, Département de Biologie, 24000 El Jadida, Maroc.

2. Université Moulay ismail, Faculté des sciences, Département de Biologie, B.P. 4010 Méknès, Maroc.

3. Université Paul Sabatier, CESAC, UMR C5576 CNRS, 118 route de Narbonne, 31062 Toulouse Cedex 04, France. 


\section{Introduction}

En milieu naturel, le développement et la succession des populations phytoplanctoniques dépendent, à chaque instant, des caractéristiques abiotiques du milieu (température, lumière, nutriments...), des compétitions interspécifiques, des pertes par sénescence, de la sédimentation et de la pression de prédation dont elles sont l'objet (Capblancq 1982, Reynolds 1984, Coesel \& Wardenaar 1994). L'apport excessif des nutriments reliés aux activités humaines est à l'origine de l'eutrophisation des écosystèmes aquatiques dont une manifestation est la prolifération des cyanobactéries. La présence de souches toxiques peut perturber l'équilibre trophique et a des conséquences sur les utilisations potentielles de l'eau:

- Colmatage rapide des filtres au niveau des prises d'eau destinées à l'adduction publique.

- Odeur et goût désagréable de l'eau (Skulberg et al. 1984).

- Sécrétion de substances toxiques qui provoque en plus de l'allergie chez les baigneurs des cas inquiétants de troubles hépatiques et intestinaux et même parfois des mortalités humaines (Jochimsen et al. 1998).

Les travaux consacrés à l'étude écophysiologique du phytoplancton ont montré qu'il existe des interactions complexes entre lumière, température d'une part, teneurs et biodisponibilité en nutriments d'autre part (Steeman-Nielsen \& Jorgensen 1968) sur la croissance algale. Lorsque les éléments nutritifs sont présents en excès, la croissance devient une fonction de la lumière et de la température dont les effets écologiques sont liés (Wetzel 1983). Par ailleurs, en cas de déficience en nutriments (azote et phosphore), les algues se développent sur leurs réserves intracellulaires "phénomène de consommation de luxe". Les travaux concernant les. capacités de stockage chez les Cyanobactéries restent très fragmentaires (Dauta 1982, Piquemal 1990, Boumnich 1992, Sbiyyaa et al. 1998).

Microcystis aeruginosa Kütz, espèce ubiquiste, se développe d'une manière subite et forme des efflorescences dans de nombreux lacs et réservoirs Marocains (Oudra 1990, Cherifi 1992, Derraz 1995).

Parmi les hypothèses émises quant aux facteurs favorisant la prolifération de ces cyanobactéries dans les lacs eutrophes et hypereutrophes, citons :

- la température élevée de l'eau (Hammer 1964, Tilman et al. 1986, McQueen \& Lean 1987);

- la faible intensité lumineuse (Zevenboom \& Mur 1980, Smith 1986);
- l'excès d'apports en nutriments (Ryther 1955, Fogg 1969).

Ce travail vise à déterminer certains paramètres écophysiologiques de Microcystis aeruginosa (taux de croissance maximal, intensité lumineuse et température optimales de croissance), les capacités de stockage en nutriments (azote et phosphore), et le suivi de la croissance en conditions nutritionnelles non limitantes ou carences en azote et phosphore.

\section{Matériel et méthodes}

Microcystis aeruginosa Kütz a été isolée de la retenue El Kansera ( $34^{\circ} 00^{\prime} \mathrm{N},-5^{\circ} 55^{\prime} \mathrm{W}$ ). Elle représente avec Oscillatoria sp. la quasi totalité de la biomasse lors des fleurs d'eaux observées dans cet écosystème à partir de 1990 (Derraz 1995, Boumnich \& Derraz, données non publiées).

Le milieu de culture utilisé est celui proposé par Dauta (1982). Totalement minéral, il évite toute prolifération bactérienne et son équilibre chimique permet des cultures de longue durée. Afin de prévenir un appauvrissement du milieu en $\mathrm{CO}_{2}$, les cultures sont fortement bullées $\left(0.5 \mathrm{l}\right.$. air $\mathrm{L}^{-1}$ de culture $\left.\mathrm{mn}^{-1}\right)$ avec de l'air filtré insufflé à la base des flacons.

La souche mère est maintenue en phase exponentielle de croissance par des repiquages fréquents ( 2 à $3 j$ ) dans un milieu riche en nutriments et dans des conditions héliothermiques suboptimales. L'éclairement est donné selon un cycle 15/9 (15 h. de lumière pour $9 \mathrm{~h}$. d'obscurité) par des tubes fluorescents blancs (type cool white) ou par des lampes PhytoClaude de 400 watts. La mesure des lumières incidentes a été réalisée à l'aide d'un quantamètre LI.COR $185 \mathrm{~A}$.

Avant l'expérience destinée à la mesure du taux de croissance en fonction de la lumière et de la température, la culture est placée 24 heures à l'obscurité (synchronisation et non préadaptation à la lumière). Au début de l'expérience, la culture est largement diluée dans un milieu neuf pour éviter tout effet d'auto-ombrage.

Pour chaque température, la culture diluée est répartie dans une série de flacons de $150 \mathrm{ml}$ sans bullage et sous un éclairage vertical dont les intensités lumineuses sont comprises entre 5 et $500 \mu \mathrm{E} \cdot \mathrm{m}^{-2} . \mathrm{s}^{-1}$.

Le taux de croissance est déterminé à partir de la densité optique (D.O. à $750 \mathrm{~nm}$ dans une cuve de $5 \mathrm{~cm}$ ), mesurée en début du cycle lumineux $\left(\mathrm{t}_{1}\right)$ et après 24 heures d'incubations $\left(t_{2}\right)$. Le taux de croissance $(\mu)$ est calculé par la formule suivante :

$$
\mu=\operatorname{Ln}\left(A_{2} / A_{1}\right) *\left(t_{1}-t_{2}\right)^{-1}
$$


où $A_{1}$ et $A_{2}$ représentent respectivement la biomasse au temps $t_{1}$ et $t_{2}$.

Dans les conditions suboptimales de température et de lumière, le suivi de la croissance de cette espèce a été étudié en conditions nutritionnelles non limitantes et dans les cas de carences en azote et phosphore. La biomasse algale est contrôlée par des mesures de la turbidité et par le dosage de la chlorophylle a.

Pour les expériences destinées à l'étude des carences, le protocole décrit par Dauta et al. (1982), a été utilisé. Des cultures maintenues en phase de croissance exponentielle sont synchronisées ( $24 \mathrm{~h}$ à l'obscurité), puis transférées et diluées dans un milieu complet pendant 6 à $8 \mathrm{~h}$, durée supposée suffisante pour que la cyanobactérie constitue des réserves intracellulaires proches de la limite supérieure (Qmax). Les cellules sont ensuite récupérées par filtration douce sur filtre Millipore $(8 \mu \mathrm{m})$, rincées abondamment et placées dans un milieu exempt du nutriment à étudier $\left(\mathrm{t}_{0}\right)$. L'évolution de cette culture, placée dans des conditions héliothermiques suboptimales, est suivie jusqu'au moment où le taux de croissance devient nul $\left(\mathrm{t}_{1}\right)$, pour des réserves intracellulaires qui tendent vers le minimum $Q_{0}\left(Q_{0}=\right.$ Quota de subsistance). Ensuite, cette même culture diluée dans un milieu neuf, est répartie dans une série de flacons de $250 \mathrm{ml}$ auxquels des doses croissantes en nutriment à étudier (azote ou phosphore apportés respectivement sous forme de $\mathrm{KNO}_{3}$ et $\mathrm{K}_{2} \mathrm{HPO}_{4}$ ) sont ajoutées.

Ces apports provoquent une reprise de croissance qui s'annule de nouveau au temps $t_{2}$. Si l'on exprime par $\mathrm{N}_{0}, \mathrm{~N}_{1}$ et $\mathrm{N}_{2}$ respectivement le nombre de cellules aux temps $t_{0}, t_{1}$ et $t_{2}$ et par. $S$ la quantité du nutriment ajouté, les stades successifs de l'expérience sont décrits par les relations suivantes:

$$
\begin{array}{lc}
\mathrm{N}_{0} * \mathrm{Qmax}=\mathrm{N}_{1} * \mathrm{Q}_{0} \text { d'où } & \mathrm{Qmax}=\left(\mathrm{N}_{1} / \mathrm{N}_{0}\right) * \mathrm{Q}_{0} \\
\mathrm{~N}_{1} * \mathrm{Q}_{0}+\mathrm{S}=\mathrm{N}_{2} * \mathrm{Q}_{0} \text { d'où } & \mathrm{Q}_{0}=\mathrm{S} /\left(\mathrm{N}_{2}-\mathrm{N}_{1}\right)
\end{array}
$$

Parallèlement à l'estimation des stocks cellulaires en nutriments, la variation du quota en chlorophylle a a été mesurée. Le dosage des pigments chlorophylliens a été réalisé selon le protocole préconisé par Marker et al. (1980).

En ce qui concerne les dénombrements cellulaires, une relation entre densité optique et nombre de cellules a été établie par des comptages au microscope inversé selon la méthode d'Utermöhl (1958); les colonies de Microcystis aeruginosa sont dissociées d'après la technique préconisée par Humphries et al. (1979).

\section{Résultats et discussion}

La figure 1 reporte les taux de croissance enregistrés à 4 températures $\left(15,20,25\right.$ et $\left.30^{\circ} \mathrm{C}\right)$ et sous des intensités lumineuses comprises entre 5 et $500 \mu \mathrm{E} \cdot \mathrm{m}^{-2} . \mathrm{s}^{-1}$. Le taux maximal de croissance obtenu est de $0.92 . \mathrm{j}^{-1}$ à $20^{\circ} \mathrm{C}\left( \pm 1^{\circ} \mathrm{C}\right)$ et une intensité lumineuse de 120 $\mu \mathrm{E} \cdot \mathrm{m}^{-2} \cdot \mathrm{s}^{-1}$. Cette valeur est très proche de celles obtenues par Mac Lachlan \& Gorham (1961) (1.04. $\mathrm{j}^{-1}$ à $23^{\circ} \mathrm{C}$ et sous une lumière continue) et par Gothan $\&$ Rhee (1981) (1.15. $\mathrm{j}^{-1}$ à $20^{\circ} \mathrm{C}$ et une intensité lumineuse de $\left.104 \mu \mathrm{E} \cdot \mathrm{m}^{-2} . \mathrm{s}^{-1}\right)$. Ce résultat demeure nettement supérieur aux valeurs $0.60 . \mathrm{j}^{-1}$ et $0.62 . \mathrm{j}^{-1}$ reportées respectivement par Okada et al. (1982) et Boumnich (1992) pour des souches isolées de lacs eutrophes tempérés. Par ailleurs, ce taux de croissance est largement inférieur aux données obtenues par Oh \& Rhee (1991) (1.79. $\left.\mathrm{j}^{-1}\right)$ et par Derraz (1995) $\left(1.28 . \mathrm{j}^{-1}\right.$ à $\left.28^{\circ} \mathrm{C}\right)$. Cette dernière valeur a été obtenue, avec bullage, pour cette souche de Microcystis aeruginosa isolée du même milieu.
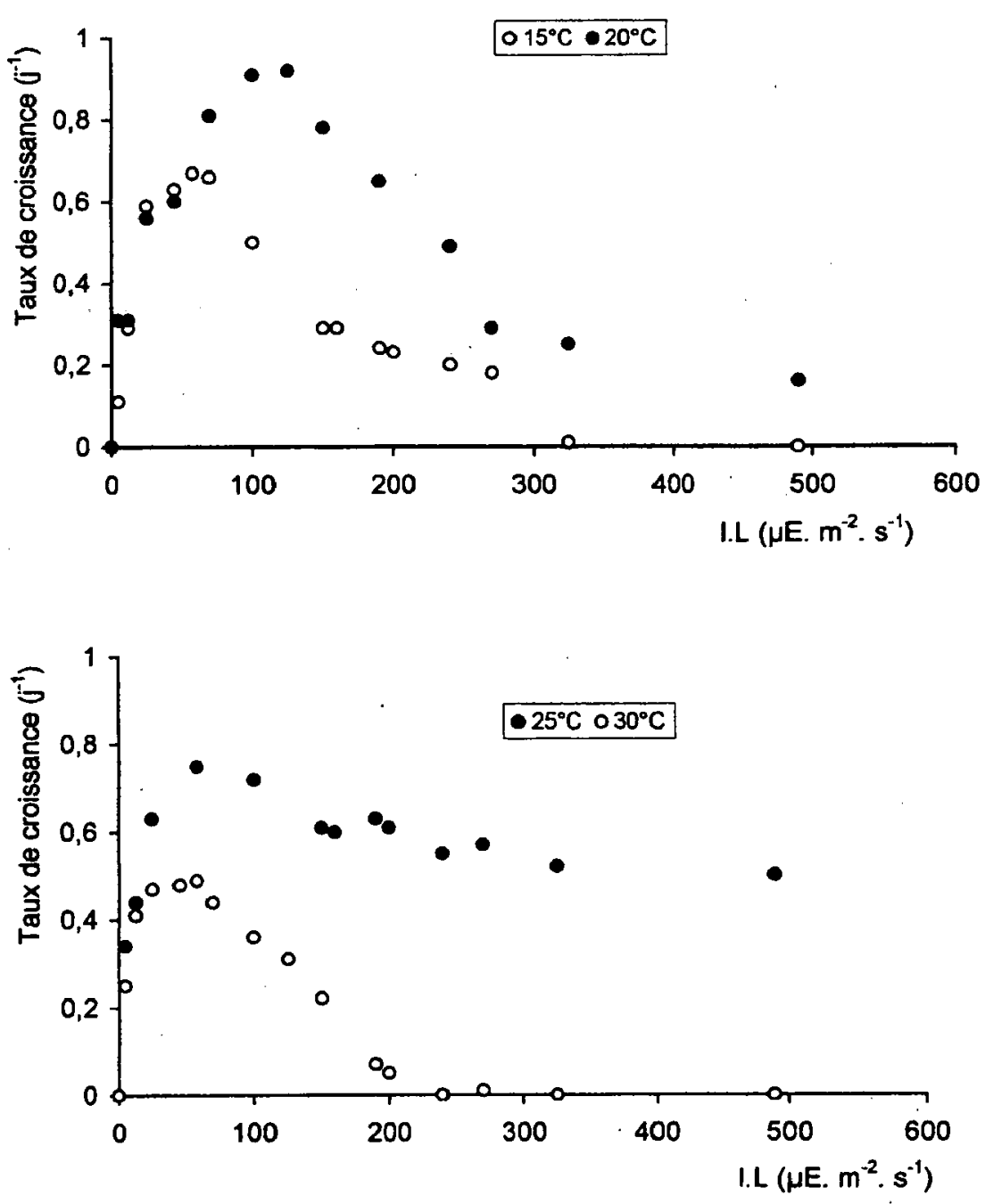

Fig. 1. Interaction de la lumière et de la température sur le taux de croissance de Microcystis aeruginosa.

Fig. 1. Interaction of light and temperature on the growth rate of $\mathrm{Mi}$ crocystis aeruginosa. 
Pour chaque température, il est possible de définir un taux de croissance optimal $\left(\mu o p t_{T}\right)$ associé à une intensité lumineuse optimale $\left(\mathrm{Iopt}_{\mathrm{T}}\right)$. Iopt $_{\mathrm{T}}$ et $\mu_{\mathrm{opt}} \mathrm{T}_{\mathrm{T}}$ subissent la même variation en fonction de la température (Fig. 2). Cette variation de Iopt $_{\mathrm{T}}$ est attribuée en particulier, au pouvoir d'adaptation de certaines espèces d'algues aux diverses intensités lumineuses (Jorgensen 1969) ; ainsi Raps et al. (1983), Paerl et al. (1985) ont montré les capacités d'adaptation de certaines souches de Microcystis aux fortes intensités lumineuses.
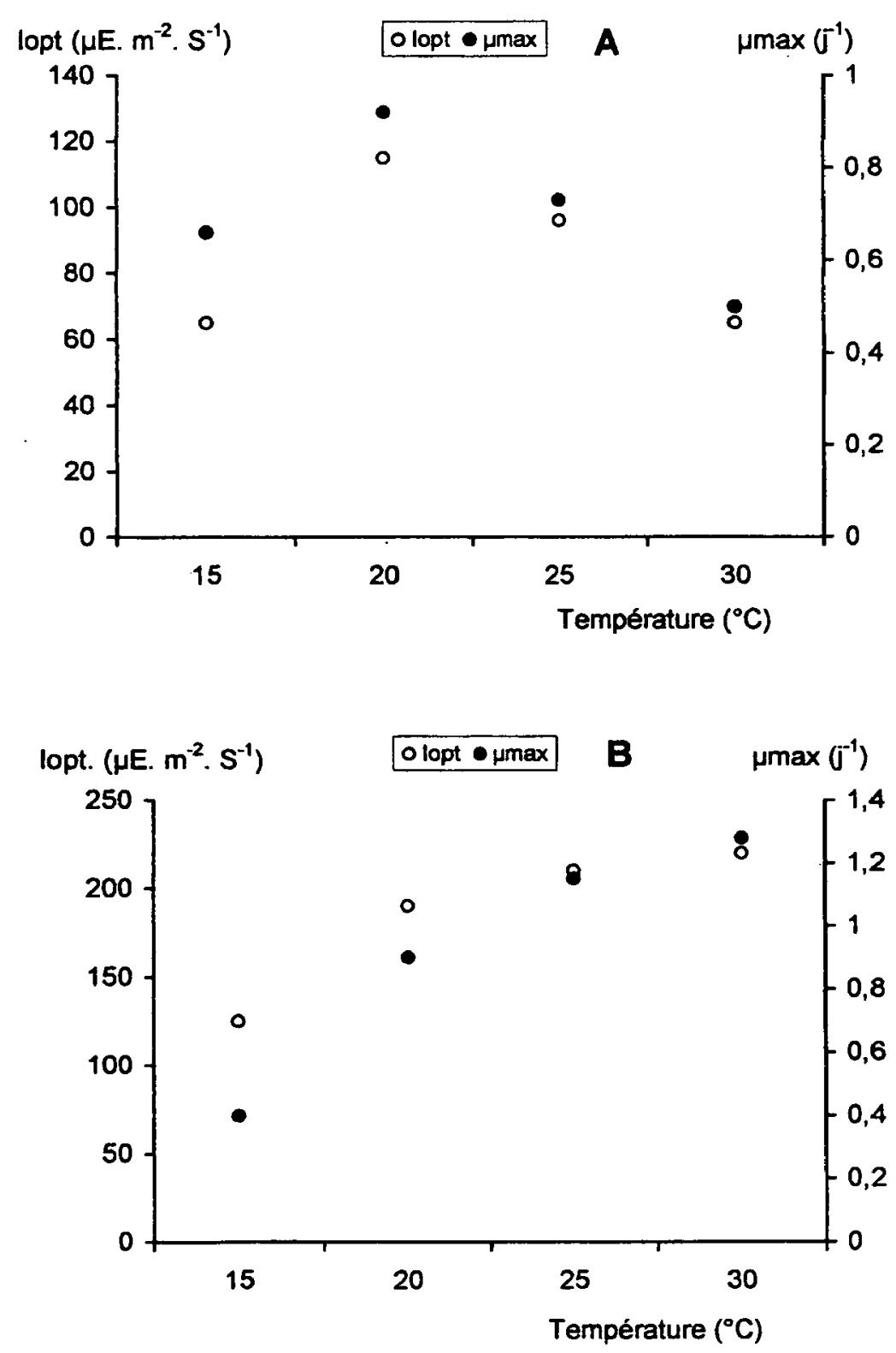

Fig. 2. Variations des paramètres de croissance $\mu$ max et Iopt de $M i$ crocystis aeruginosa en fonction de la température : (A) sans bullage ; (B) avec bullage (Derraz 1992).

Fig. 2. Growth constants $\mu$ max and Iopt for Microcystis aeruginosa, as function of temperature : (A) without bubbling ; (B) with air bubbling (Derraz 1992).
La croissance des algues est également sous la dépendance de la température (Goldman \& Carpenter 1974, Goldman 1977, Ahlgren 1987). De nombreux auteurs (Krüger \& Eloff 1978, Foy \& Gibson, 1982, Foy 1983, Reynolds 1984, Dauta et al.1990, Oh \& Rhee 1991, Boumnich 1992, Derraz 1995) ont montré que l'optimum thermique pour le développement des cyanobactéries est compris entre 25 et $37^{\circ} \mathrm{C}$. La valeur de l'optimum thermique déduit de notre expérience, réalisée sans bullage, reste légèrement inférieure $\left(20^{\circ} \mathrm{C}\right)$.

Pour le suivi de la croissance de Microcystis aeruginosa (Fig. $3 \mathrm{~A}$ ) dans des conditions nutritionnelles non limitantes, les deux paramètres utilisés pour l'estimation de la biomasse (densité optique et chlorophylle a) évoluent dans le même sens et montrent une corrélation positive $\left(\mathrm{r}^{2}=98 \%\right.$ ) (Fig. $3 \mathrm{~B}$ ). A partir du 11 ème jour suivant la mise en culture, les teneurs en chlorophylle a subissent une légère chute qui pourrait être associée à l'effet d'auto-ombrage. En revanche dans les cas de carences, la biomasse algale produite sur les réserves intracellulaires reste faible (Fig. $3 \mathrm{C}$ et D). Un arrêt de la croissance se produit dès le 4 ème jour pour la culture privée d'azote et le $8^{\text {ème jour pour }}$ celle privée de phosphore. Quant aux variations des teneurs en chlorophylle a, elles ne reflètent pas l'évolution de la biomasse et se trouvent beaucoup plus affectées dans les cas des carences en azote qu'en phosphore.

Les figures 4 et 5 montrent que les modifications cellulaires conséquentes à la déficience en éléments nutritifs (azote et phosphore) se traduisent par une réduction des teneurs en chlorophylle plus importante dans le cas de carence en azote qu'en phosphore (il n'y a plus de relation entre biomasse et chlorophylle).

Privées de source d'azote ou de phosphore, les cellules se multiplient en utilisant leurs réserves, les stocks intracellulaires évoluent des quotas maximums $\left(Q_{\operatorname{maxN}}\right.$ et $\left.\mathrm{Q}_{\max \mathrm{P}}\right)$ vers les quotas minimums $\left(\mathrm{Q}_{\mathrm{ON}}\right.$, $\mathrm{Q}_{\mathrm{OP}}$ ). Les valeurs des quotas cellulaires obtenues (Tableau 1) montre que Microcystis aeruginosa stocke 5.33 plus de phosphore que d'azote $\left(\mathrm{Q}_{\max \mathrm{P}} / \mathrm{Q}_{\mathrm{oP}}\right) /$ $\left(\mathrm{Q}_{\operatorname{maxN}} / \mathrm{Q}_{\mathrm{oN}}\right)$.

\section{Discussion}

Ces paramètres de croissance et de stockage des éléments nutritifs de Microcystis aeruginosa, isolée d'un lac de barrage eutrophe au Maroc, constituent des données nouvelle utiles à la compréhension des capacités d'adaptation de cette espèce aux conditions environnementales. Le taux spécifique de croissance maximale obtenu s'intègre dans l'intervalle des résultats rappor- 

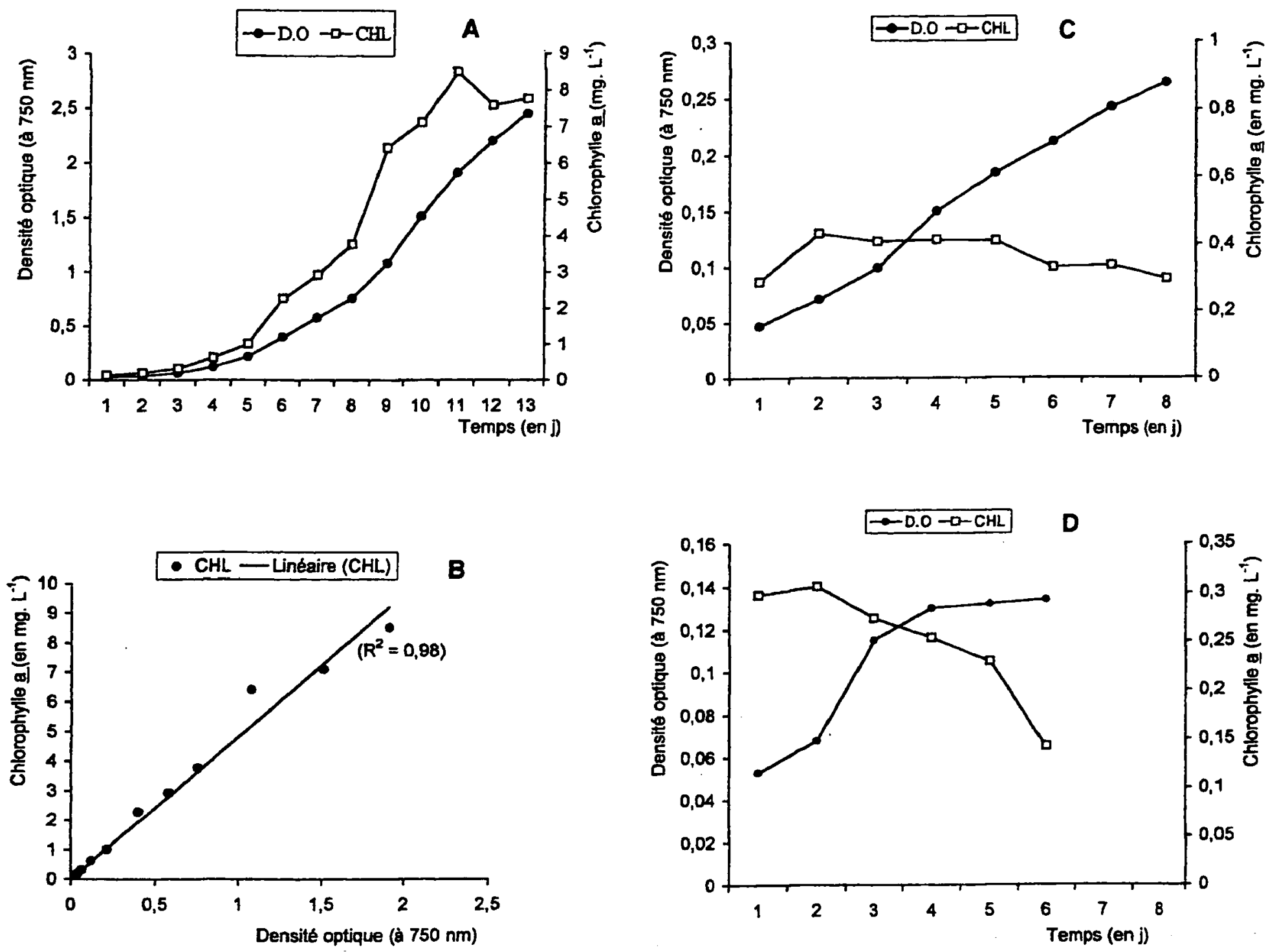

Fig. 3. Suivi de la croissance de Microcystis aeruginosa. Sur milieu complet (A), corrélation entre la teneur en chlorophylle a et la densité optique (B), milieu sans azote (C), et milieu sans phosphore (D).

Fig. 3. Growth rate of Microcystis aeruginosa. Complete medium (A), correlation between chlorophyll a and optic density (B), medium without nitrogen (C), and medium without phosphorus (D).

Tableau 1. Données de la littérature relatives au stockage intracellulaire de l'azote et du phosphore pour des souches de Microcystis aeruginosa.

Table 1. Literature data relative to intracellular nịtrogen and phosphorus storage for Microcystis aeruginosa.

\begin{tabular}{lcccccr}
\hline \multirow{2}{*}{ Références } & \multicolumn{3}{c}{ Azote $\left(\mu \mathrm{g}\right.$ at. $10^{9}$ cell $\left.^{-1}\right)$} & \multicolumn{3}{c}{ Phosphore $\left(\mu \mathrm{g}\right.$ at. $10^{9}$ cell $\left.^{-1}\right)$} \\
& $\mathrm{Q}_{0}$ & $\mathrm{Q}_{\mathrm{m}}$ & $\mathrm{Q}_{\mathrm{m}} / \mathrm{Q}_{\mathrm{o}}$ & $\mathrm{Q}_{\mathrm{o}}$ & $\mathrm{Q}_{\mathrm{m}}$ & $\mathrm{Q}_{\mathrm{m}} / \mathrm{Q}_{0}$ \\
\hline Boumnich 1992 & 189 & 356 & 1.88 & 4.64 & 31.32 & 6.75 \\
Sbiyyaa et al. 1998 & 20.5 & 49.17 & 2.40 & 0.91 & 11.61 & 12.76 \\
Sbiyyaa et al. 1998 & 23.58 & 56.14 & 2.38 & 1.12 & 13.76 & 12.28 \\
Souche étudiée & 29.86 & $\mathbf{7 5 . 8 4}$ & $\mathbf{2 . 5 4}$ & $\mathbf{1 . 3 5}$ & $\mathbf{1 8 . 2 8}$ & $\mathbf{1 3 . 5 4}$ \\
\hline
\end{tabular}



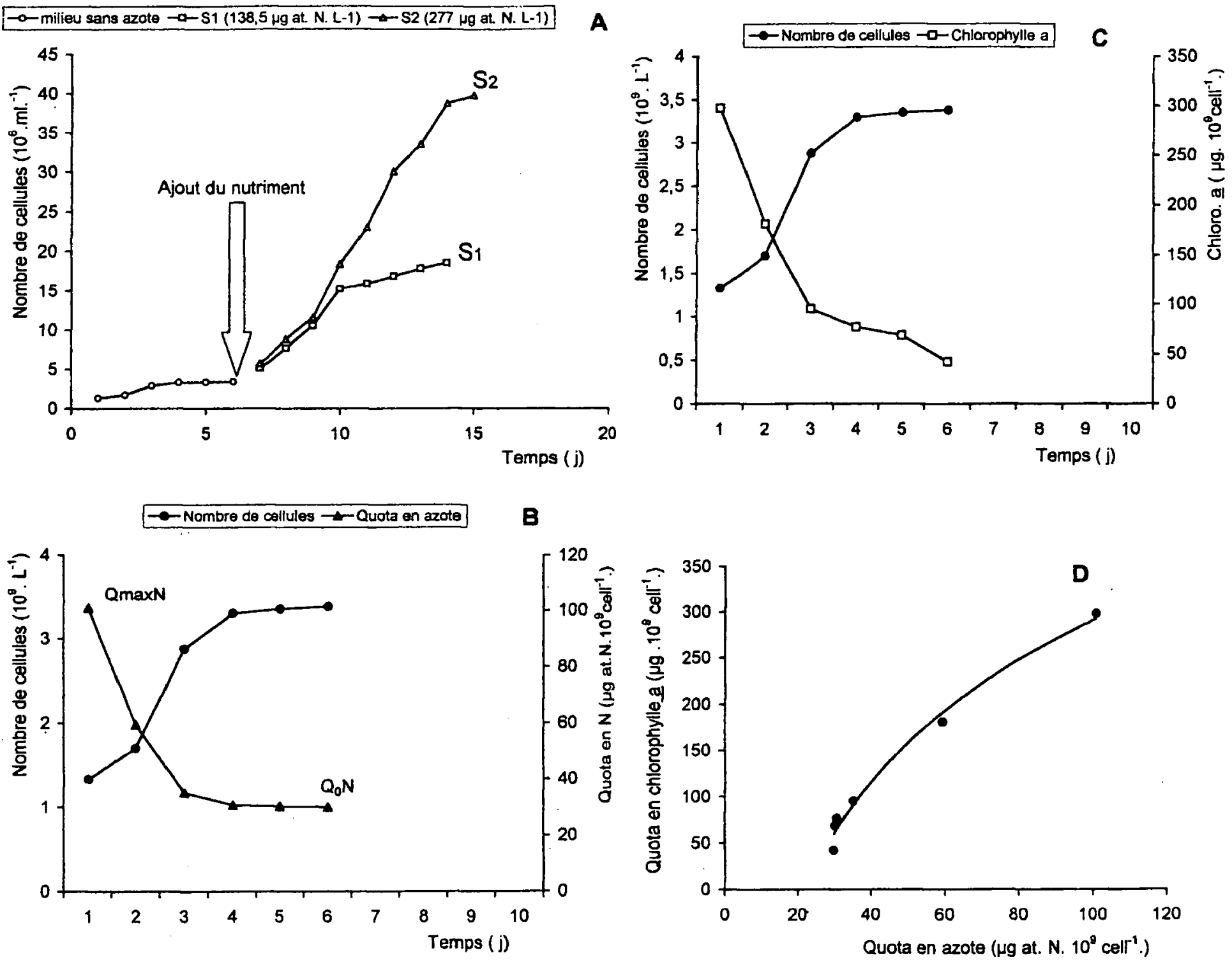

Fig. 4. Carence en azote chez Microcystis aeruginosa. Estimation des quotas internes (A), variation du nombre de cellules et du quota en azote au cours de la carence (B), variation du nombre de cellules et du quota è chlorophylle a au cours de la carence (C), et relation entre réserves intracellulaires en nutriments et la teneur des cellules en chlorophylle a (D).

Fig. 4. Estimation of internal nitrogen quota for Microcystis aeruginosa (A), variations of number of cell and nitrogen quota in the course of the deficiency (B), variations of number of cell and chlorophyll quota in the course of the deficiency (C), and relationship between internal nitrogen quota and chlorophyll quota of cell (D).

tées par Dauta (1982) et Zohary (1985), selon lesquels, le taux de croissance des cyanobactéries est généralement compris entre 0.4 et $1 . \mathrm{j}^{-1}$. L'importante variabilité des valeurs recensées dans la littérature pour ce paramètre, en fonction de la lumière et de la température confirme l'hypothèse communément émise selon laquelle, il existerait un nombre considérable de souches de Microcystis aeruginosa de caractères métaboliques extrêmement variés (Carmichael \& Gorham 1981, Krüger \& Eloff 1981, Nicklisch et al. 1983).

En dehors des conditions de carence en nutriments, la densité optique et la chlorophylle a sont bien corrélées. Leur mesure présente l'avantage de donner un résultat rapide et significatif pour l'estimation de la biomasse.
Cette cyanobactérie est capable de stocker beaucoup plus de phosphore que d'azote; ainsi chaque cellule permet la production de 13 cellules filles contre 2 à 3 pour les réserves azotées. Le quota minimal pour chaque nutriment $\left(Q_{0} j\right)$ est une constante spécifique, indépendante des paramètres héliothermiques, dont la valeur pour chaque élément est atteinte après des durées variables de carence (Fuhs 1969). Le coefficient de "consommation de luxe" $\left(\mathrm{Q}_{\max } / \mathrm{Q}_{0}\right)$ est une constante spécifique (Tilman \& Kilhan 1976, Lang \& Brown 1981) qui permet d'évaluer la capacité de stockage et la compétitivité de l'espèce vis-à-vis de l'utilisation du nutriment considéré. Les teneurs en chlorophylle a sont beaucoup plus affectées dans le cas de déficit en azote qu'en phosphore. 

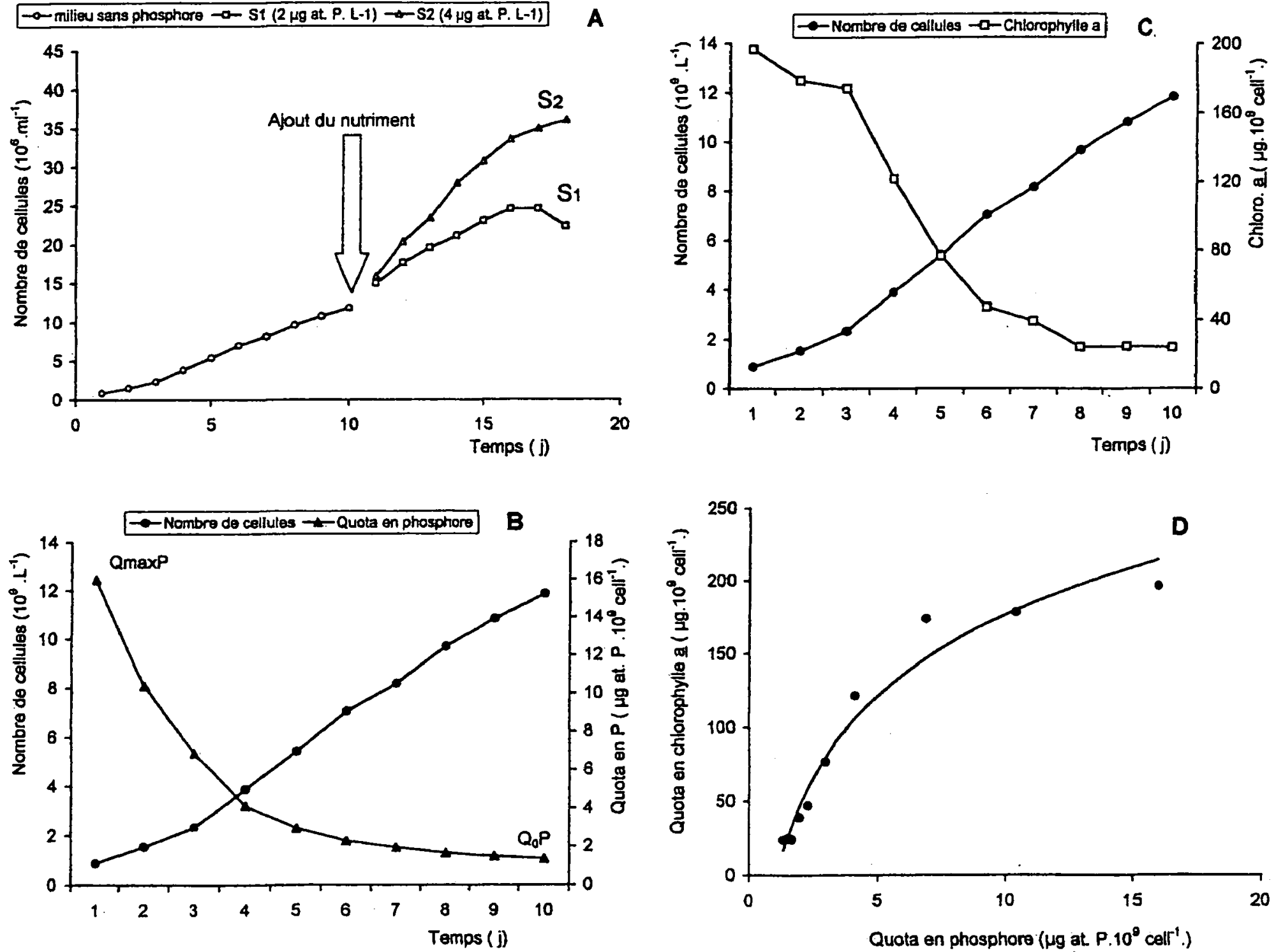

Fig. 5. Carence en phosphore chez Microcystis aeruginosa : Estimation des quotas internes (A); variations de nombre de cellules et du quota en phosphore au cours de la carence (B), variations de nombre de cellules et du quota en chlorophylle a au cours de la carence (C), et relation entre réserves intracellulaires en nutriments et la teneur des cellules en chlorophylle a (D).

Fig. 5. Estimation of internal phosphorus quota for Microcystis aeruginosa (A), variations of number of cell and phosphorus quota in the course of the deficiency (B), variations of number of cell and chlorophyll quota in the course of the deficiency (C), and relationship between internal phosphorus quota and chlorophyll quota of cell (D).

\section{Remerciements}

Ce travail a été effectué avec le support financier du projet européen INCO DC ERB. 3514 IC18-CT98-0293.

\section{Travaux cités}

Ahlgren G. 1987. - Temperature functions in biology and their application to algal growth constants. Oikos, $49: 177-190$.

Boumnich L. 1992. - Etude expérimentale des paramètres écophysiologiques des principales espèces phytoplanctoniques du lac d'Aydat (Puy-de-Dôme, France). Essai de modélisation des cultures d'algues. Thèse Doct. Univ., Univ. Blaise Pascal, ClermontFerrand II : $172 \mathrm{p}$.

Boumnich L., Dauta A., Devaux J.\& Romagoux J.C. 1990. - Influence de la lumière et de la température sur la croissance de quatre espèces d'algues d'un lac eutrophe (Lac D'Aydat Puy-deDôme, France). Ann. Limnol., 26 (1) : 3-10.

Capblancq J. 1982. - Phytoplancton et production primaire. In Ecologie du plancton des eaux continentales. Pourriot $\mathrm{R}$. (ed.) Masson, Paris : 1-48.
Carmichael W.W. \& Gorham P.R. 1981. - The mosaic nature of toxic blooms of cyanobacteria. In The water environment : algal toxins and health. W.W. Carmichael (ed) : 161-172.

Cherifi O. 1992. - Evolution ét dynamique du phytoplancton en relation avec certains paramètres biotiques et abiotiques de la retenue Lalla Takerkoust. Thèse $3^{\text {eme }}$ cycle , Univ. Cadi Ayyad, Faculté des Sciences Semlalia, Marrakech: 155 p.

Coesel P.F.M. \& Wardenaar K. 1994. - Light-limited growth and photosynthetic characteristics of tow planktonic desmid species. Freshwater Biol., 31 : 221-226.

Dauta A. 1982. - Conditions de développement du phytoplancton: Etude comparative du comportement de huit espèces en culture. II- Rôle des nutriments : assimilation et stockage intracellulaire. Ann. Limnol., 18 (3) : 263-292.

Dauta A. 1982. - Conditions de développement du phytoplancton : Etude comparative du comportement de huit espèces en culture. I. Détermination des paramètres de croissance en fonction de la lumière et de la température. Ann. Limnol., 18 : 217-262. 
Dauta A., Brunel L. \& Guerri M.M. 1982. - Détermination expérimentale des paramètres liés à l'assimilation de l'azote et du phosphore par Scenedesmus crassus. Ann. Limnol., 18 (1) : 33-40.

Dauta A., Devaux J., Piquemal F. \& Boumnich L. 1990. - Growth rate of four freshwater algae in relation to light and temperature. Hydrobiologia, 207 ; 221-226.

Derraz M. 1995. - Etude de l'eutrophisation de la retenue du barrage El Kansera (Maroc) : caractéristiques physico-chimiques, biodisponibilité du phosphate sédimentaire, écophysiologie de $\mathrm{Mi}$ crocystis et relations des blooms avec les paramètres environnementaux. Thèse d'Etat, Univ. Moulay Ismail : $119 \mathrm{p}$.

Fogg G.E. 1969. - The physiology of an algal nuisance. Proc. $R$. Soc. London, ser. B. $173: 175-187$.

Foy R.H. 1983. - Interaction of temperature and light on the growth rates of two planktonic Oscillatoria species under a short photoperiod regime. Br. Phycol. J., $18: 267-273$.

Foy R.H. \& Gibson C.E. 1982. - Photosynthetic characteristics of planktonic blue-green algae : The response of twenty strains grown under high and low light. Br. Phycol. J., 17 : 169-182.

Fuhs G.W. 1969. - Phosphorus content and rate of growth in the diatoms. Cyclotella nana and Thalassiosira fluviatilis. J. Phycol., $5: 312-321$.

Goldman J.C. 1977. - Temperature effects on phytoplankton growth continuous culture. Limnol. Oceanogr., 22 (5) : 932-936.

Goldman J.C. \& Carpenter E.J. 1974. - A kinetic approach to the effect temperature on algal growth. Limnol. Oceanogr., 19 (5) : 756-766.

Gothan I.J. \& Rhee G.Y. 1981. - Comparative kinetic studies of phosphate-limited growth and phosphate uptake in phytoplankton in continuous culture. J. Phycol., $17: 257-265$.

Hammer V.T. 1964. - The succession of 'bloom' species of bluegreen algae and some causal factors. Verh. Int. Ver. Limnol., 15 829-836.

Humphries S.C. \& Widjaja F. 1979. - A simple method for separating cells of Microcystis aeruginosa for counting. Br. Phycol. J., 14 (4) : 313-316.

Jochimsen E.M. 1998. - Liver failure and death after exposure to microcystns at a hemodialysis center in Brazil. The New England Journal of Medecine, Vol. 38 (13) : 873- 878

Jorgensen E.G.1969. - The adaptation of plankton algae. IV. Light adaptation in different algal species. Physiol. Plant., $22(6)$ : 1307-1315.

Krüger G.H.J. \& Eloff J.N. 1978. - The effect of temperature on specific growth rate and activation energy of Microcystis and Synecoccus isolates, relevant to the onset of natural blooms. J. Limnol. Soc. S. Afr., 4 (1) : 9-20.

Krüger G.H.J. \& Eloff J.N. 1981 - - The effect of physico-chimical factors or growth relevant to the mass culture of axenic Microcysti..In The water environment: : Algal toxins and health Carmichael W.W (ed) : 193-222.

Lang D.S. \& Brown E.J 1981. - Phosphorus limited growth of green algae and blue-green algae. Appl. Envirnm. Microbio., 42 (6) : 1003-1009.

Marker A.F.H., Nash E.A. \& Riemann B. 1980. - The measurements of photosynthetic pigments in freshwater and standardisation of methods : conclusions and considerations. Ergebn. Limnol., $14: 91-106$

McLachlan J. \& Gorham P.R. 1961. - Growth of Microcystis aeruginosa Kütz. In a precipitate free medium buffered with Tris. Can. J. Microbiol., 7 : 869-882.

McQueen D.J. \& D.R.S. Lean 1987. - Influence of water temperature and nitrogen to phosphorus ratio on the dominance of bluegreen algae in Lake St. George, Ontario. Can. J. Fish. Aquat.Sci., $44: 598-604$.
Nicklisch A. \& Kohl J.G. 1983. — Growth kinetics Microcystis aeruginosa Kütz as a basis for modelling its population dynamics. Int. Revue Ges. Hydrobiol., 68 (3) : 317-326.

Oh H.M. \& Rhee G.Y. 1991. - A comparative study microalgae isolated from flooded rice paddies: light-limited growth, $\mathrm{C}$ fixation, growth efficiency and relative $\mathrm{N}$ and $\mathrm{P}$ requirement. Journal of applied Phycol., $3: 211-220$.

Okada M., Sudo R. \& Aiba S. 1982. - Phosphorus uptake and growth of blue-green algae Microcystis aeruginosa. Biotechnol. Bioeng., 24 : 143-152.

Oudra B. 1990. - Bassins de stabilisation anaerobie et aerobie facultatif pour le traitement des eaux usées à Marrakech. Dynamique du phytoplancton (Microplancton et picoplancton) et éva-

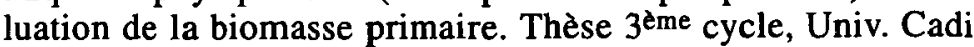
Ayyad, Faculté des Sciences Semlalia, Marrakech : 144 p.

Paerl H.W., Bland P.T., Bowles N.D. \& Haibach M.E. 1985. Adaptation to light intensity, low-wavelenght light among surface blooms of the cyanobacterium Microcystis aeruginosa. Appl. Environ. Microbiol., 49 : 1046-1052.

Piquemal F. 1990. - Contribution à l'étude écophysiologique de quelques espèces d'algues en culture. Thèse Doctorat, Univ. Paul Sabatier, Toulouse III : 137p.

Raps S., Wyman K., Siegelman H.W. \& Falkowski P.G. 1983. Adaptation of the cyanobacterium Microcystis aeruginosa to light intensity. Plant Physiol., 72 : 829-832.

Reynolds C.S. 1984. - The ecology of freshwater phytoplankton, Cambridge University Press, London : 384 p.

Ryther J.H. 1955. - Ecology of autotrophic marine dinoflagellates with reference to red water conditions. In The luminescence of biological systems : Johnson F.H. (ed.) : 387-414.

Sbiyyaa B., Loudiki M. \& Oudra B. 1998. - Capacité de stockage intracellulaire de l'azote et du phosphore chez Microcystis aeruginosa Kütz. Et Synechocystis sp. : cyanobactéries toxiques occasionnant des blooms dans la région de Marrakech (Maroc). Ann. Limnol., 34 (3) : 247-257.

Skulberg O.M. and al. 1984. - Toxic blue-green algal blooms in Europe: A growing problem. Ambio, 8 (4) : 244- 247.

Smith V.H. 1986. - Light and nutrient effects on the relative biomass of blue-green algae in lake phytoplankton. Can: J. Fish. Aquat. Sci., 43 : 148-153.

Steeman-Nielsen E. \& Jorgensen E.G. 1968. - The adaptation of plankton algae. I. General. Part. Physiol. plant., 21 : 401-413.

Tilman D. \& Kilhan S.S. 1976. - Phosphate and silicate growth and uptake kinetics of the diatoms Asterionella formosa and Cyclotella meneghiniana in batch and semicontinuous cultures. J. Phycol., $12: 375-383$.

Tilman D., Kiesling R, Sterner R., S.S. Kilhan \& F. A. - Johnson 1986.- Green, blue-green, and diatom algae : Taxonomic differences in competitive ability for phosphorus, silicon, and nitrogen. Arch. Hydrobiol., 106 : 473-485.

UtermöhlV.H. 1958. - Zür Vervollkommung der quantitative Phytoplankton Metodik. Mitt. Intern. Verein. Theor. Angew. Limnol., $9: 1 \_38$.

Wetzel R.G. 1983. - Limnology. W.B. Saunders Campany publishing, Philadelphia : $767 \mathrm{p}$.

Zevenboom W. \& L.R. Mur 1980. $-\mathrm{N}_{2}$-fixing cyanobacteria : Why do they not become dominant in Dutch. Hypertrophic Lakes. In Hypertrophic Ecosystems Barica J. \& L.R. Mur (eds). Dr W. Junk Publishers, The Hague : 123-130.

Zohary T. 1985. - Hypersecums of the cyanobacterium Microcystis aeruginosa in hypertrophic lake (Hartbeesport Dam. South. Africa). J. Plankton Res., 7 (3) : 399-406. 